\title{
Common Causes for Dissatisfaction of International Patients and Remedial Measures With Respect to Indian Legal System
}

\author{
C. L Avadhani $1^{\text {* }}$ \\ ${ }^{1}$ B.Sc, AMIE, MBA, M. Phil, MMM, PGDFM, PGDMM, PGDBA, PGDHRM, PGDPM\&IR, PGDIPR, DLL, LLM, Research Scholar, (Ph. D), \\ Annamalai University, Tamil Nadu, India
}

Article History

Received: 09.03.2021

Accepted: 14.04.2021

Published: 24.04.2021

Journal homepage: https://www.easpublisher.com

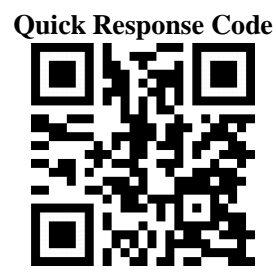

Abstract: Globalization of healthcare opening up of economy entry of private players into the healthcare sector, has brought in see saw change globally. Many developing nations are fast emerging as global healthcare players to attract International Patients. India is emerging as a leading destination for healthcare procedures / interventions to International Patients with cost effective procedures zero or less waiting time high quality procedures with latest technology and highly skilled professionals that are in comparison with developed nations. However, healthcare sector is dealing with human feelings and humans there will be always dissatisfaction because the patients expectations and want needs may not be attended to by the caregivers of the destination country. In this article a detailed discussion is made out regarding patient's dissatisfaction at various stages and at various circumstances from the time of admission till the time of discharge, in addition the causes of dissatisfaction, remedial measures, how to satisfy the want needs of the patient are all discussed with appropriate examples. In addition the legal situation and also the laws to attract such discomfort and dissatisfaction of the patients and the remedial measures are also discussed elaborately.

Keywords: Dissatisfaction, legal rights, want needs, destination country, professionals attitude.

Copyright (C) 2020 The Author(s): This is an open-access article distributed under the terms of the Creative Commons Attribution 4.0 International License (CC BY-NC 4.0) which permits unrestricted use, distribution, and reproduction in any medium for non-commercial use provided the original author and source are credited.

\section{INTRODUCTION}

Globalization of healthcare, opening up of economy, many private players entering into the healthcare sector, there is a see saw change with respect to healthcare globally. Many developing nations such as India, Malaysia, Singapore, Thailand are emerging as best competitive players in healthcare compared to developed nations like U.S., U.K., Germany etc.,This change has brought in as a boon to the health sector in developing nations as mentioned above and also a boon to the International Patients who are otherwise deprived of their expectations such as prohibitive cost, waiting time in their own country. Hence, many International Patients are opting for healthcare procedures / interventions in India because they can get their ailments / discomfort attended to at affordable cost with less or zero waiting time and procedures and interventions that are comparable to advanced countries and are not available in their home country. However, this in flow of International Patients are also having its own negative aspects such as dissatisfaction of the patients that are to be attended, to satisfy the want needs of the International Patients.
WHAT IS PATIENT DISSATISFACTION? [2]

Dissatisfaction is a negative aspect of patient satisfaction. International Patients seek overseas healthcare with some pre conceived notions/ expectations and if their expectations are not fulfilled then they get dissatisfied. In addition if an International Patient's want need with respect to their illness / discomfort is not attended to their expectation then the patient gets dissatisfied.

Following are some of the factors are contributing to the patient dissatisfaction,

1) Non procedural dissatisfaction at different levels from admission to discharge:

Non procedural dissatisfaction starts with the arrival of the International Patient to the healthcare facility, of the destination country.

a) Admission: When an international Patient arrives to the healthcare facility of the destination country, the patient should be guided properly to the International Help Desk otherwise if the process is cumbersome the patient gets dissatisfied. The patient starts thinking that his choice of healthcare facility has gone wrong. 
b) International Patient's Help Desk: Since the International patient's are coming from globally some may not have knowledge of other languages even English except their mother tongue. If such patients are not given proper attention to clear their doubts in their mother tongue or the language they know the patient gets dissatisfied because if the help desk speaks in a language not comfortable to the patient, the patient will not get the true picture and the details he wanted. This causes an embrassment not only to the help desk but also to the patient and the dissatisfaction crops in.

c) Appointment with Healthcare Professionals in initial stage: Unless the International Patient Help Desk guides the patient to the proper professionals either pre appointed or otherwise and if the professionals have not taken proper action in meeting the patient's requirements dissatisfaction will start.

d) Pre procedural examinations such as tests (X-ray, MRI, CT Scan etc., ) and checking the health records that are available with the patient and personal examination of the patient by the professionalsby interviewing the patients and their accomplice to get more information regarding patients ailments / discomfort to the patient satisfaction. This meeting should be in a cordial manner and the healthcare professional who is handling the patient should take all the precautions by attending all the queries raised by the patients and their accomplice with patience. Sometimes, the doubts and expectations raisedby the patient or accomplice may be unusual but the healthcare professional should clear all their doubts with ease and clarity.

e) Subsequently, the healthcare professional who is attending the patient will write some tests, for further information and to decide the type of procedure / intervention needed to the discomfort / ailment of the patient. The instructions that are given in writing should be legible, clean without any abbreviations so that while undergoing tests the patient will be in a position to know that the patient is getting the desired tests wanted by the professional. Any lapse on the part of the technician in understanding the instructions will have repercussions on the patient's discomfort and extra spending for a repeated tests and will cause dissatisfaction to the patient.

f) An Healthcare professional who is going to attend the ailment / discomfort of the patient (surgeon / physician) will go through the test reports, health records past and present and subsequent test results / reports, and will come to a conclusion the type of procedure / intervention, whether it is surgery or treatment that is needed. The professional has to inform the patient and their accomplice regarding the nature of ailment / discomfort and the proposed intervention either surgery or medicinal and the pros and cons of such intervention. The professional should clearly inform the patient regarding the ailment / discomfort and at what stage it is (initial / advanced / terminal) and the need for such intervention and approximate time within which the intervention has to be taken place otherwise the effects of non attending immediately. Here the professional also should inform the patient and their accomplice regarding time that will take to complete the procedure and approximate days the patient has to in healthcare facility and the aftermath of the procedure. At this stage, the patient gets so many doubts regarding alternative procedures/ interventions, non surgical interventions, reducing the days of hospitalization etc., and the professional should attend to all his doubts and clear them. Here the patient and the accomplice are given an approximate cost of the procedure including stay in hospital till the patient is discharged.

g) The administrative staff who are responsible for attending non procedural service such as admission, discharge, billing etc., should clear all the doubts of the patient and their accomplice and in addition they should enquire whether the patient is an insured one or not.

h) If the patient is an insured and whether the insurance policy mentions the clause of payment (prepayment, post payment, partial payment) and accordingly they should give the estimate to the patient for their acceptance.

i) The other point being flexibility in foreign exchange rates (Rs vs. Patients Currency) and the administrative staff should make it clear the procedure they adopting regarding foreign exchange and payment of fee.

j) The hospital administration should give them the choice of hospital environment (room for the patient and accomplice) and cost involved facilities available and additional facilities in accordance with patient's desires and wishes on extra charges. If these things are not informed and a consent is obtained there will be a grudge at the time of billing and the patient will be dissatisfied.

k) During discharge and at the time of final billing the administration should keep in mind the understanding between the patient and the authorities at the time of admission and the terms and conditions. Here also, if the details are not given by the different departments from the hospital before during and after procedure and also the medicines, medical equipment, surgical ancillaries that are drawn from the pharmacy in the name of the patient and if the unused items are not returned back the cost of them may appear in the billing causing dissatisfaction. Hence it is the responsibility of the patient and the authorities to check whether such items are included or excluded in the bill.

1) Finally, at the time of discharge the patient should be given a format that includes, the kind of medication required subsequent and after discharge, the precautions that are needed, the food 
and other intakes that are prohibited / used should be given in writing by the healthcare professional. In addition a details guidelines should also be given during travel by the patient till he reaches home and the care that is needed during travel. Even after reaching the home the necessary care the patient has to take and if required how to contact the professionals who attended in India their mail address, telephone numbers, probable times of attending should also be clearly mentioned in the discharge report. If any of these things are not attended by the caregivers the patient gets dissatisfied.

2) Procedural Dissatisfaction $[24,25]$ :

a) Once a decision is taken, regarding the procedure / intervention / surgery that is needed to contain if not eliminated the disease / discomfort of the patient, the procedural dissatisfaction will come into play. As already mentioned above, the tests, reports are not properly made the patient has to go for additional tests causing dissatisfaction both mentally and monetarily.

b) Before procedure the anesthetist should have a consultation and appraising the situation with the patient and accomplice. The anesthest should inform the patient the type of anesthesia that is given during procedure (partial, full, local or total) and also the time of procedure and the time required to the patient to come to the conscious after the procedure. Here, the anesthetist should examine and take cognizance of the patient's present condition, the ailment, the procedure, the approximate time for the procedure and also the medicines that are allergetic to the patient; also he/she should get information from the patient, his/her food habits (vegetarian / Non vegetarian) whether he/she is tea totallar or not or any other habits he/she is having. A thorough discussion is necessary because the anesthesia depends on all these factors. Any wrong or missed fact will become fatal at times. Hence, the patient and their accomplice should give true picture regarding the patient's habits and any items that are allergic and the anestheist should be careful enough in choosing the type of anesthesia.

c) During procedure, sometimes unexpected complications may arise knowingly or unknowingly and the professionals who are attending should take immediate steps else, the situation may go out of hands. Here, the consent may not be possible but the professional should take all necessary care and follow the established and approved norms during such critical situations. This abnormal situation may burn the pocket of the patient, and the professional should explain the patient and their accomplice the reasons for such situation and how it was handled to save the life of the patient and bring the patient to normalsy. If proper explanation is not given by the professional the patient get dissatisfied. d) Normally after the procedure the patient is kept in Intensive Care Unit till he/she comes out of the anesthesia. Here the ICU staff (nurses and paramedical staff) should be very careful in treating the patient because the patient is in unconscious state not knowing what he/she needs, and the nurses should keep a $24 \mathrm{Hrs}$ watch and attend to the patient all the needs that are instructed by the professionals till the patient comes to the conscious.

\section{3) During Hospitalization [26, 28]:}

When an International patient or in fact any patient is admitted for a procedure or intervention to their ailment/discomfort will have numerous problems because the environment in the hospital is not akin to them. The movement the patient is hospitalized the patient is having tremendous amount of stress and anxiety because the patient is very much worried regarding his/her ailment / discomfort and the procedure / intervention and the outcome. Psychologically the patient will not be in a position to subject to procedure / intervention because the patient always thinks whether the procedure / intervention is successful or not and that he/she will come out free from discomfort.

a) Role of healthcare professionals: It is the responsibility of the healthcare professional / specialist who is treating the patient to prepare the patient for the procedure and make him feel comfortable leaving all the doubts aside. Otherwise, the patient will have anxiety and tension that will reflect on the health and procedure and recovery.

b) Environment: Environment both internal and external should be conducive to patient as well as employees. A positive healthcare facility / environment makes employees feel good about coming to work and provide the motivation to sustain them throughout and thereby give best of their care to the patient to their satisfaction, thus eliminating any element of dissatisfaction in the patient's mind [22].

c) Healing Environment: Healthcare buildings describe a physical setting and organization culture that supports the patients and their accomplice through the stress imposed by illness, hospitalization, medical visits, the process of healing and sometimes bereavement. The concept is that physical healthcare environment can make a difference how quickly the patient recovers or adopts to specific acute and chronic conditions. This kind of environment is to reduce stress and thereby reduce associated problems such as, medical error, inability to concentrate and physical symptoms of stress of the professionals that can affect logical thought and process, thereby dissatisfying the patient [22].

d) Bringing hope to the healthcare facility: The fact that the patient has to be in the healthcare facility for several days and at times even one's because of 
their illness / discomfort, creating healthcare environment should make not only the patient but also their loved one's feel like that they can relax and may be even forget that they are the healthcare facility [22]

e) Importance of Lighting and Sound: $80 \%$ of what we interpret of our surroundings comes to us from what we see offer environment and that is greatly affected by the light available in the facility. Lighting design in healthcare facility is a major factor in creating healing situations. It is a proven fact that people who are surrounded by natural light are more productive and live healthier hence it is important for the providers to see every room to have a window for natural light to come into and help create a healing environment to the patient thereby minimizing the dissatisfaction [22].

f) Sound: Nightingale the lady of the lamp claimed that,

"sounds that create anticipation, expectation, waiting and fear of surprise... damaging the patient " [22]

She also mentions,

"variety, color and form as a means of arising creativity and help in patients"

According to the, "business case for creating a healing environment", written by Jain Malkin,

The physical setting has the potential to be therapeutic if it achieves the following.

(i) Eliminates environmental stresses such as noise, glare, lack of privacy, and poor air quality.

(ii) Connects patient's to nature with views to the outdoors, interior gardens, aquariums, water elements etc.,

(iii) Offers options and choices to enhance feeling of being in control. These may include privacy vs. socialization, lighting level, type of music, treating options, quiet vs. active, mating areas

(iv) Provides opportunities for social support seating arrangements that provides privacy for family groupings, accommodation for family members in treatment settings; sleep - over accommodation in patient's rooms causes dissatisfaction.

(v) Provides positive distraction such as interactive art, fire places, internet connections, music, access to special video programs with soothing images of nature accompanied by music developed specially for the healthcare settings and finally

(vi) Engenders feelings of peace, hope, reflection and spiritual connection and provides opportunities for relaxation, education, human and Vinci.

Another exponent of healthcare, Lucy Maddox, questions [21]

Can the way hospitals are designed improve the experiences of staff and visitors and even the recovery of the patients.
Alain D Botton mentions,

If one room can alter, how we feel, if our happiness can hang on the colour of the walls or the shape of a floor what will happen to us.In most of the places, we are forced to look at and inhabit? What will be experience in a house in a prison like windows, stained carpet, tiles and plastic curtains?

g) Washrooms: It is very important that washrooms used by the patients should be clean and make them disinfectant else, it becomes the cause of infectious diseases to the patients. Hence, to satisfy the patients and to eliminate the additional discomfort, the washrooms, corridors all should be taken care of. Covid-19 pandemic has exposed the sanitary conditions in every healthcare facility and the loop holes patient's to express their unwillingness to join the healthcare facilities.In this regard the Government of India has declared safety measures that are to be implemented immediately by healthcare facilities so that no patient will be affected by any infectious disease including Covid19. Also, the Government of India made it clear that hospitals that are treating Covid-19 patients should be separated with the rest of the facilities so that the Pandemic will not spread.

h) Paramedical Staff: The present alarmic situation of Covid-19 Pandemic and the second wave rapidly spreading in the country all the paramedical staff and testing facilities and such other important functions in the hospital where the procedures and tests are conducted should be sanitized frequently without harming the patients nor making them feel discomfort / dissatisfied.

i) Diagnostic Errors: When a patient is admitted to healthcare facility, the professional examine the patient to diagnose the ailment / discomfort through the existing health records, personal observation and conversation with the patient. In this process there is possibility of committing diagnostic errors and sometimes these errors will become fatal. The common diagnostic errors are [16, 23]:

i. Missed Diagnosis,

ii. Wrong Diagnosis,

iii. Delayed Diagnosis

iv. Failure to recognize the complications, means, identifies right diagnosis but could not identified the complications, during or after procedure

v. Failure of diagnosing related disease, complications, during or after procedure

vi. Failure to diagnose unrelated diseases

If the patient is affected with anyone of these errors they can claim compensation and criminal proceedings on the professionals and the providers under criminal, civil and consumer courts, including vicarious liability.

j) Medical Negligence [10, 13-15]: According to Winfield, 
Negligence is defined as, the breach of a legal duty, to take care of a person who is suffering from illness results in damage, undesired by the defendant to the plaintiff [2].

He also stated that,

Negligence has three essential components,

Duty, Breach and Resulting Damage

It is the breach of the duty to care meaning, thereby carelessness in a matter related in which the law mandates carefulness. A breach of this duty gives a patient the right to initiate action against negligence.

The Apex Court in Paramanda KataraVs. Union of India (1989) observed,

Every Doctor whether Government Hospital or otherwise has the professional obligation to extend his / her services for protecting life. The obligation being total, absolute and paramount, loss of procedure whether in statues or otherwise cannot be sustained and therefore must give way.

The same Apex Court in another Judgment in Indian Medical Association Vs. Santha held that [4, 9],

Doctor who rendered service free of charge in a hospital are not covered under the provisions of Consumer Protection Act, as their services "service as defined under Sec. 1(o) of the Act." only those doctors and nursing home / hospitals come under this act which charges fee for their services. Any minimal amount is being charged as registered fee is not fee.

Hence, any negligence on the part of the healthcare professional, paramedical staff, nurses causing dissatisfaction / harm to the patient at times, becomes fatal will come under civil, criminal and vicarious liabilities.

k) Communication: The most important aspect and the cause of dissatisfaction at every stage in an healthcare facility right from admission till discharge is improper / lack of communication / low communication [20, 26, 27].

Communication, is a process of conveying meaning from one person to another in the broadest sense it refers to the whole process of man's life in relation to the group [19].

For a planning to be realistic and to remove dissatisfaction in the minds of a patient it is essential that it should be based on sound information, which is not possible without a good system of communication. The main function of communication is interaction between patient, accomplice, healthcare professionals, nurses, paramedical staff and other administrative staff connected with the facility.

The patient always expects courtesy and respect from the healthcare professionals and others in the healthcare facility but any lapses in the communication will have adverse impact and the patient get dissatisfied. Communication is of different types such as verbal, written, signs and such other modes that conveys a word from one person to other. In healthcare a body language is very important to healthcare professionals when they deal with the patient as such they should keep in mind "open body posture" and face the patient while communicating. There are many barriers to effective communication between healthcare professionals and patients, according to Prof. Ofri.

Many research studies have shown that good communication with healthcare professionals and the patients have many benefits and the patients will be more satisfied.

(i) It greatly contributes to the ability to provide patients with individual care

(ii) The healthcare professionals, more so the nurses, who take the time to understand the unique challenges and anxieties of their patients should have better communication skills so that they can address the problems of the patient and dispel their fear and dissatisfaction.

(iii) This attitude of the professionals will make the patients feel that they are receiving the best attention from the professionals, thereby they can express their feelings, symptoms, quicker and better

(iv) Interpersonal communication can satisfy the want needs of the patient according to Maslow's hierarchy of needs.

\section{l) Misguidance by facilitators to attract International Patients to the facility of their choice:}

Unlike other travel arrangements healthcare arrangements are different because in healthcare travel, people travel to other than own, to receive healthcare procedures and the travel is case specific. The Travel Arrangements will have different packages in line with the patient's want needs. If an International Patient is coming to the destination country to a facilitator, the patient is circumvened by the facilitator's choice of facility and package, there is always dissatisfaction when the patient comes to know that there are other facilitators who can give better service.

m) Attitude towards patients by [19],

a) Doctors /specialists / duty doctors

b) Paramedical staff

c) Nursing staff and attendants

d) Lab Technicians

e) Sweepers and other class-iv employees

Here the above mentioned persons attitude towards International Patients is most important because any lapses in the part of above personal will cause dissatisfaction. If a duty Doctor or Specialist do not attend a patient in emergency and any delay will have dissatisfaction. Likewise, the nurses and the 
paramedical staff if they won't attend the patient to satisfy their want needs especially during medication and feeling discomfort of sleep or to attend Nature's call, this dissatisfaction will be maximum likewise, if the patient is taken to tests or laboratories or X-rays and CT Scans any delay are improper handling of the patient, the patient gets dissatisfied and even at times make complaints.

\section{n) Loss of dignity and privacy [20]:}

Any patient who is not given proper dignity irrespective of his classification will have bad effect on their satisfaction. The patient will always feel dignified in their own way and they expect similar respect in the healthcare facility.

The other important aspect is privacy, every patient feel that their ailment/discomfort and nature of treatment should be kept isolated and the patient will not allow except the professionals who are attending to know their ailment / discomfort. Hence it is the duty of the caregivers to give utmost privacy to every patient in handling the cases. A recent example in a multi specialty hospital, a chief executive of a multinational company has come for checkup. While he was setting in the visitors lounge nurse has come and started taking blood sample. This annoyed the patient and he left the facility because his privacy was not guaranteed.

o) Commercialization leads to lack of human touch and human feeling: [20]

It is a known fact that private healthcare facilities are commercial establishments and they want profits for their investment. Hence, the caregivers / healthcare providers tries to introduce technology so that they can reduce man power. Here the professionals will depend mainly on the results obtained instead of examining the patient personally except in few cases. This kind of technological innovation and intervention leads to lack of human touch and human feeling. The patient will never have any close contact with the professionals to went their feelings and grievances.

4) Post Procedural Dissatisfaction during and after discharge

When the patient medically recovers in accordance with the expectations of the healthcare professionals who attended will inform the patient about their discharge that means, the patient has recovered to the extent that he can travel to his home country.

It is a common cause of dissatisfaction for International Patients at the time of discharge when they look at the billing. It becomes very disheartening when they come with a medical package either through a facilitator or directly to the healthcare facility under an agreed healthcare package. If the patient does not discuss all the relevant details that are included and excluded from the package and any other incidental / accidental costs that may crop in during discharge.
Otherwise, there will be a dissatisfaction and at times leads to arguments and chaois.

Following are some of the unforeseen circumstances that will make the bill at the time of discharge more than expectation / package:

1. Unexpected complication arising during procedure that makes the caregivers to go beyond the prescribed procedure which will costs more in terms of additional procedure, medication and service provided including any equipment.

2. Unexpected complications arising due to non availability / non providing of past health records by the patient "

3. Longer stay than expected

4. Recuperation / Rehabilitation after procedure in certain ailments thatare necessary, which are not included in the package offered by facilitator / provider.

5. The other important aspect is drugs that are needed and medical ancillaries that are drawn from the pharmacy of the healthcare facility in the account of patient and if unused items are not returned, there is every possibility of costs of these items are included in the billing, causing dissatisfaction and frustration to the patient.

6. Additional Charges that come in the way of getting specialized personal to the need of the patient from other sources in emergency cases will have frustration to the patient.

7. At times, over cautious precautionary measures of the healthcare professionals will make the patient to undergo some additional tests, MRI scanning, Doupler tests etc., will be charged additionally.

8. During the process blood requirements / platelets vary according to the procedural situation that are made available extra will cause more in the billing.

9. Different insurance policies will have frustration to the patientsif they are not co-related with the rules and regulations of the healthcare facility.

10. Last but not the least due to oversight or otherwise, arithmetical mistakes if they are not properly checked will cause dissatisfaction and patient start arguing with the concerned personal.

\section{LEGAL RECOURSE}

Indian Laws and Acts that attracts healthcare cases

a) Civil Jurisdiction $[1,5,6]$

b) Criminal Jurisdiction [3, 7, 8, 10-12]

c) Consumer Protection Act as amended from time to time [9]

d) Information Technology Act, 2000 (I.T. Act, 2000 - with latest amendments)

e) Information Technology (Reasonable security, practices and procedures and sensitive personal data or information) Rules, 2011 (I.T. Rules, 2011)

f) Indian Healthcare Council (Professional Conduct Etiquette and Ethics) Regulations, 2002 - (MCA Code of Ethics)

g) Healthcare Termination of Pregnancy Act, 1971 
h) The Human Immunodeficiency virus and acquired immune deficiency syndrome (Prevention and Control) Bill, 2014

i) Statements as defined by the Private Securities Litigation Reform Act, 1997

j) New Anti Corruption Law creates changes to wisitle blowers - Lexology (Registration)

k) Art Bill may close surrogacy doors for foreigners, unmarried people

1) Drugs and cosmetic act and cosmetic rules, 1945

\section{Redressal Mechanism for International Patient's Rights:}

1. Disputes resolution through Public Dialogues and Public hearings, issues, outcome and follow

2. Grievance Redressal through in house complaints forums at the institutional level

3. Cause of action for complaints related to health before designated District Courts
a. Remedies
b. Orders of designated District Courts
c. Reasoned Order
d. Costs
e. Binding effect
f. Consequences of breach of designated courts

Temporary orders

\section{g. Appeals}

h. Time frame for designation of courts

i. dispensing of lawyer's appearance and waiver of court fee

\section{j. Information on website}

4. Enforcement of monetary orders,

a. recovery of compensation costs awarded to

b. insurance cover by private establishments

5. Residuary offences, penalties and Immunities or Criminal Penalties

\section{Conclusion}

Healthcare sector, is a sensitive sector wherein human lives, feelings are involved. Unlike other service sector wherein any loss of service will have only financial repercussions but healthcare is not the same. Any lapses by anybody / any event / any circumstances will be fatal at times. Hence, the healthcare professionals and all the caregivers should be very careful in their planning procedure and implementation. Any lapses on their part leads to dissatisfaction to the patient and their accomplice that varies according to the situation and circumstances. A patient will come to the healthcare facility thinking that his/ her ailment / discomfort will be attended to by the professionals and that the patient gets himself / herself relieved from such ailment / discomfort and goes to attend their normal work. As already mentioned above there are various causes / circumstances both procedural and non procedural that will come in the way of patient dissatisfaction.

Due to Covid-19 Pandemic the central government of India has restricted the International
Patient's movements through travel restrictions to different parts of the globe. In addition the Government announced that only one attendant will be allowed along with a patient coming from overseas and both of them should be kept in quarantine for 14 days before they are allowed to any healthcare facility. This will have greater dissatisfaction to the patient as they have to wait for 14 days before getting any healthcare. In addition there are many lockdowns in many parts of the country. The international patient should choose the facility where such restrictions are not there. That means, the patients choice of facility is limited and has no choice except to accept the facility that is available (dissatisfaction). Presently, the fast increasing of second wave of pandemic covid-19 has still made the International Patients have their procedures / interventions overseas, has become more difficult or even remote. Hence, it is the prerogative of caregivers to see that these dissatisfactions are minimized if not eliminated so that the patient will leave the facility satisfied if not fully satisfied.

\section{REFERENCES}

1. Poonam Verma v. Ashwin Patel \&Ors. - SCC

2. Black's Law Dictionary

3. Bhalchandra @ Bapu\& Another v. State of Maharashtra - SCC

4. Jacob Mathew v. State of Punjab \& Another - SCC

5. AchutraoHaribhauKhodwa\& Others v. State of Maharashtra \& Others - SCC

6. C.P. Sreekumar (Dr.), MS (Ortho) v. S. Ramanujam - SCC

7. Kurban Hussein Mohammed ali Rangawalla v. State of Maharashtra - SCC

8. Sir Lawrence Jenkins in Emperor v. OmkarRampratap

9. Indian Medical Association Vs..V.P. Shantha\& Others - SCC

10. Mohan Vs. Prabhaji Nair and another (2004) CPJ 21 (SC)

11. Hampton Vs State: State Vs Lester

12. ShivaramVs the State, AIR 1965, All 196

13. Martin D Souza Vs Mohammad Isfaq (2009) 2 SCC 40

14. Vijay Sinha Roy (D) by LR Vs. Biswanath Das

15. AnuradhaSaha Vs. AMRI Hospital

16. N. Shrikhandevs Anita Sena Fernandes [(2011) 1 SCC 5

17. Bombai Mirror, Nov.22, 2016)

18. Jaipur Golden Hospital, Rohini, Delhi. (India Times)

19. https://online.regiscollege.edu/blog/7-tipsensuring-patient-safety-healthcare-settings/

20. https://blog.cureatr.com/improve-patientsatisfaction-with-a-focus-on-patient-safety

21. https://www.psqh.com/mayjun08/satisfaction.html\# : :text=Thus\%2C\%20patients\%20associate\%20saf ety $\% 20$ and,satisfaction $\% 20$ and $\% 20$ reduced $\% 20$ ad verse $\% 20$ events 
22. https://www.ahrq.gov/patientsconsumers/diagnosis-treatment/hospitalsclinics/10-tips/index.html

23. https://en.wikipedia.org/wiki/Patient_safety

24. https://pssjournal.biomedcentral.com/

25. https://www.rasmussen.edu/degrees/healthsciences/blog/patient-safety-in-hospitals

26. https://www.hsj.gr/medicine/the-development-ofpatient-safety- culture.php?aid=3262\#: :text=The\%20factors $\% 20$ involved\%20in\%20the,done\%20on\%20patient $\% 20$ safety $\% 20$ culture.

27. https://www.cureatr.com/patient-safety-bridgingthe-information-gap-cureatr

28. https://www.ncbi.nlm.nih.gov/pmc/articles/PMC30 57365/

Cite This Article: C. L Avadhani (2021). Common Causes for Dissatisfaction of International Patients and Remedial Measures With Respect to Indian Legal System. East African Scholars Multidiscip Bull, 4(3), 14-21. 